\title{
Use of isotope scanning as an aid to the diagnosis of pulmonary embolism in the accident and emergency department
}

\author{
M. REICHL, I. S. BENTLEY \& R. A. SLEET \\ Accident and Emergency Department, Southampton General Hospital, Southampton, \\ England
}

\section{SUMMARY}

Twenty-five patients with a clinically suggestive diagnosis of pulmonary embolism were referred directly from the accident and emergency department for a ventilation and perfusion isotope scan. On the basis of a negative scan, the authors were able to discharge 19 patients. The advantages of having direct access to isotope imaging are described.

\section{INTRODUCTION}

Many patients present to the accident and emergency (A\&E) department complaining of pleuritic chest pain. The diagnosis is often clinically obvious. However, there is a group of patients in whom pulmonary embolism (PE) cannot be excluded on clinical grounds (Wenger et al., 1972). Despite the lack of firm diagnosis, such patients are frequently admitted and anticoagulated (Robin, 1977). The diagnositic accuracy can be significantly increased using isotope lung scanning (Fulkerson et al., 1986). For some time, it has been the authors' practice to refer patients with a clinical diagnosis of PE, presenting to the $A \& E$ department during working hours, for an immediate ventilation perfusion $(\mathrm{V} / \mathrm{Q})$ isotope lung scan. In this paper, they retrospectively review their experience with direct access to $\mathrm{V} / \mathrm{Q}$ scanning. 


\section{METHOD}

During a one-year period, 25 patients were referred directly from the A\&E department $\frac{3}{\Phi}$ for an isotope lung scan. These patients presented with either pleuritic chest pain or $\stackrel{?}{-}$ with chest pain shortness of breath and risk factors for PE. However embolism could $\overrightarrow{\vec{F}}$ not be excluded on clinical grounds. There were 13 men and 12 women. The mean age was 41.5 years (range 19-71). All the patients had chest X-rays. Routing isotope imaging was carried out using a gamma scintillation camera without quantification.

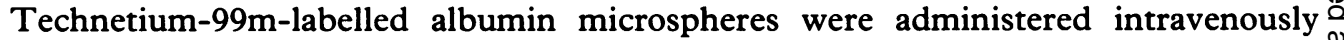
with the patient supine. Krypton-81m in oxygen was breathed from arubidium- $81^{\circ}$ generator (MRC cyclotron unit, Hammersmith Hospital, London, England) (Fazio \& $\vec{\circ}$ Jones, 1975). Patients were imaged in the anterior, posterior and both posterior oblique $\vec{\omega}$ positions. Each view was measured alternately with the gamma camera setting at $\frac{\Omega}{D}$ $140 \mathrm{kev}$ and $190 \mathrm{kev}$ with no change in the position of the patient. Images were 3 produced on photographic film.

\section{RESULTS}

The presenting symptoms and signs are listed in Table 1.

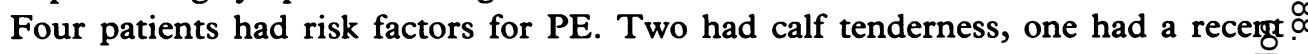
operation and one had a previous PE. None of these patients were eventually diagnosel by the lung scan as having PE. Each patient had a chest X-ray. Six radiographs we
abnormal.

The abnormalities found were: two instances of partial lobar collapse, one of $\frac{\varrho}{\mathcal{Q}}$

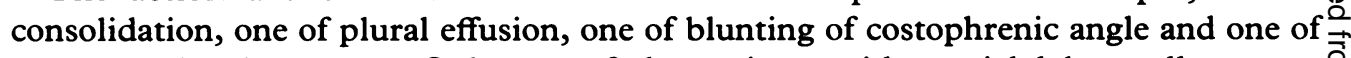
hilar gland enlargement. Only one of the patients with partial lobar collapse was $\frac{\hat{3}}{3}$ eventually shown to have PE by the $\mathrm{V} / \mathrm{Q}$ scan. The majority of the patients with $\mathrm{PE}$ had a normal chest X-ray.

All the patients were referred for a V/Q scan. This was performed within $3 \mathrm{~h}$ of their arrival in the A\&E department. Five out of the 25 scans were abnormal. Four were interpreted as showing PE and one was compatible with chest infection. The other 203. scans were normal. These results are summarized in Fig. 1.

Table 1 Symptoms and signs in the patients with and without PE

\begin{tabular}{|c|c|c|c|c|c|}
\hline Symptoms & PE & No PE & Signs & PE & No PE \\
\hline Pleuritic chest pain & 3 & 14 & Nil & 0 & 12 \\
\hline Chest pain & 1 & 6 & $\begin{array}{l}\text { Tachycardia } \\
(>90 \text { beats } / \mathrm{min})\end{array}$ & 2 & 8 \\
\hline Shortness of breath & 1 & 4 & Crepitations & 3 & 4 \\
\hline Cough & 1 & 2 & Tender chest & 1 & 4 \\
\hline Collapse & 0 & 1 & Reduced air entry & 1 & 3 \\
\hline \multirow[t]{2}{*}{ Haemoptysis } & 0 & 1 & Pleural rub & 1 & 1 \\
\hline & & & Calf tenderness & 0 & 2 \\
\hline
\end{tabular}




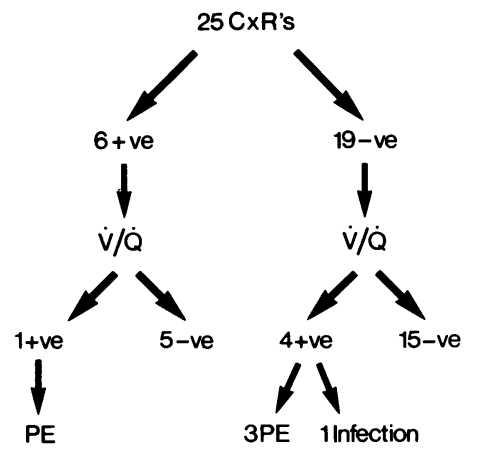

Fig. 1 Summary of the results of chest X-rays (CXR) and V/Q scans.

Of the 25 patients, six were admitted. Of these, four were anticoagulated on the basis of their abnormal $\mathrm{V} / \mathrm{Q}$ scans. The other two were admitted for further investigations. Neither was shown to have PE. Nineteen patients were discharged home. None of them died or were admitted in the subsequent 2 months with a PE.

\section{DISCUSSION}

There are many patients presenting to the $A \& E$ department who are relatively well and haemodynamically stable, but have symptoms suggestive of PE. In many, it proves difficult to make a firm clinical diagnosis of PE because the symptoms and signs of PE are non-specific (Wenger et al., 1972; Guidotti et al., 1979). This is well demonstrated in the 25 patients from our department. All of them complained of chest pain and, in the majority (17), this was pleuritic. Signs of this group were varied and did not contribute greatly to the diagnosis (Table 1). In fact, one of the patients with PE had no abnormal clinical findings except a mild tachycardia ( 92 beats/min). Similarly, plain chest X-ray and laboratory investigations are frequently unhelpful (Szucs et al., 1971; Robin, 1977). Only six of our patients had an abnormal chest X-ray. However, the radiographs of three out of four patients with PE were normal.

Failure to accurately diagnose PE and start appropriate treatment can lead to increased mortality and morbidity (Fulkerson et al., 1986). Because of this, there is a tendency to over diagnose PE (Robin, 1977) and, hence, many patients are admitted and anticoagulated unnecessarily (Guidotti et al., 1979). However, anticoagulation itself carries a significant morbidity and some mortality (Mant et al., 1977). Such admissions and treatment also carry certain financial implications. PE was included in the differential diagnosis of all of the 25 patients. However, in none of them could the diagnosis be made clinically with sufficient certainty to admit them for anticoagulation.

To aid in the diagnosis of PE, patients were referred from the A\&E department for an immediate $V / Q$ scan. This was used because it is the most specific non-invasive test available (Scotsman et al., 1986). A completely normal scan virtually excludes the 
possibility of PE (McBride, 1986). Twenty of the patients had normal scans and it was possible to dismiss the diagnosis of $\mathrm{PE}$ in these patients and discharge 19 of them. None $\frac{\mathbb{D}}{6}$ of them were subsequently shown to have PE. The remaining five patients had 3 abnormal scans. Four of these were thought to be compatible with the diagnosis of PE. $\stackrel{\circ}{?}$ All these patients were admitted and anticoagulated as it was felt that the probability of $\overrightarrow{\vec{s}}$ PE was sufficiently high that they did not warrant a pulmonary arteriogram. One patient ${ }_{0}^{+}$ with abnormal scan was thought to have a chest infection, and he was started on antibiotics and discharged to his general practitioner.

Without the ready availability of isotope imaging, the majority of the patients with $\stackrel{\widetilde{\Phi}}{2}$ negative scans would have been admitted and, pending further investigations, they would have been anticoagulated. As only grossly abnormal scans confirm the diagnosis $\overrightarrow{0}$ of PE (Sharma \& McIntyre, 1984) and scans between the two extremes are far less $\overrightarrow{\vec{\omega}}$ specific (McNeil et al., 1974), many authors feel that isotope scans are not useful for the ${ }^{\omega}$ positive diagnosis of PE. Pulmonary arteriography is the most accurate investigation for $\frac{\mathbb{D}}{3}$. the diagnosis of pulmonary embolism (Windebank, 1987). As this investigation is if invasive, carries a certain degree of morbidity and cannot be performed on an outpatient ${ }_{\%}^{\mathrm{N}}$ basis, it is not applicable in the A\&E setting. However, in the A\&E department, where one is often concerned with excluding the diagnosis of $P E$, a $V / Q$ scan is a good screening test. Due to technical difficulties, many hospitals do not have access to $V / \mathrm{Q}_{\bar{C}}$ scans. Isotope perfusion scans are much more widely available. Although this type of $\frac{\sim}{\widetilde{D}}$ scan is less specific than the $V / Q$ scan, a normal result will again exclude the diagnosis of $\vec{\varphi}$ PE (Windebank, 1987). Therefore, this type of scan can be used as a screening $\infty_{\infty}^{\infty}$ procedure in the way the authors used the $V / Q$ scans.

This retrospective review shows the usefulness of having direct access to isotope $\mathrm{V} / \mathrm{Q}$ scanning from the A\&E department. The authors were able to exclude PE in 21 out $3 \mathrm{f}$ 25 patients on the basis of a normal scan. Nineteen of the patients with normal scans $\frac{0}{\varnothing}$

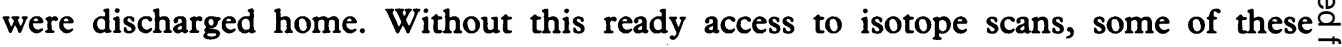
patients would have been admitted and anticoagulated. As the scan is only operational $\overrightarrow{\vec{O}}$ between the hours of 9 a.m. to 5 p.m. from Monday to Friday, patients attending outside these times are still admitted and anitcoagulated until a scan is obtained.

\section{ACKNOWLEDGEMENT}

The authors wish to thank Dr D. M. Ackery, Director of Nuclear Medicine, Southampton General Hospital, Southampton, England, for his assistance.

\section{REFERENCES}

Fazio F. \& Jones T. (1975) Assessment of regional ventilation by continuous inhalation of radioactive 0 krypton-81m. British Medical fournal iii, 673-6.

Fulkerson W. J., Coleman R. E., Ravin C. E. \& Saltzman H. A. (1986) Diagnosis of pulmonary embolism. Archives of Internal Medicine 146, 961-7.

Guidotti T. L., Friers L. F., Bell W. R., Gurley H. T. \& Pachner A. (1979) Accuracy of screening for pulmonary embolism in the emergency room. Respiration 37, 309-17. 
McBride K. (1986) Can ventilation perfusion scans accurately diagnose acute pulmonary embolism? Archives of Surgery 121, 754-7.

McNeil B. J., Holman L. \& Adelstein J. (1974) The scintigraphic definition of pulmonary embolism. fournal of the American Medical Association 227, 753-6.

Mant M. J., Thong K. L. \& Kirtwhistle R. V. (1977) Haemorhagic complications of heparin therapy, Lancet i, 113-5.

Robin E. D. (1977) Overdiagnosis and overtreatment of pulmonary embolism: the emperor may have no clothes. Annals of Internal Medicine 87, 775-87.

Scotsman H. D., Rapoport S., Gottschalk A. \& Greenspan R. H. (1986) Imaging of pulmonary embolism. Investigating Radiology 21, 443-54.

Sharma G. V. R. K. \& McIntyre K. M. (1984) Pulmonary embolism. Cardiology Clinics 2, 269-74.

Szucs M. M., Brooks H. L., Grossman W., Banas J. J., Meister S. G., Dexter L. \& Dalen J. E. (1971) Diagnostic sensitivity of laboratory findings in acute pulmonary embolism. Annals of Internal Medicine 74, $161-6$.

Wenger N. K., Stain P. D. \& Willis P. W. (1972) Massive acute pulmonary embolism: the deceivingly nonspecific manifestations. Fournal of the American Medical Association 220, 843-4.

Windebank W. J. (1987) Diagnosis of pulmonary thromboembolism. British Medical fournal 294, 1369. 